\title{
Papers
}

\section{Expression of E-cadherin in oesophageal carcinomas from the UK and China: disparities in prognostic significance}

\author{
Wei-Guo Jian, S Jane Darnton, Keely Jenner, Lucinda J Billingham, Hugoe R Matthews
}

\begin{abstract}
Aims-To study the expression and prognostic significance of the cell adhesion molecule E-cadherin in oesophageal tumours from the UK (low risk area) and China (high risk area).

Methods-E-cadherin expression was measured immunohistochemically in resected tumours from 17 patients in the UK with adenocarcinoma, 23 patients from the UK with squamous carcinoma, and 30 patients from China with squamous carcinomas who survived for five years postoperatively and compared with similar tumours from patients in the same regions who did not survive (140 tumours in
\end{abstract} all).

Results-Normal squamous epithelial cells and well differentiated areas of tumours showed membranous staining for E-cadherin expression. Cytoplasmic staining, heterogeneous staining, or an absence of staining was seen in dysplastic epithelium and in less well differentiated areas of tumours. Only one of 140 primary tumours had homogeneous membranous expression. In tumours from UK patients with adenocarcinoma $(p=1.00)$ and from Chinese patients with squamous carcinomas $(p=0.06)$ there was no correlation between $E$-cadherin absence and nonsurvival. In tumours from UK patients with squamous carcinomas there was a significant correlation between absence of E-cadherin and non-survival $(p=0.009)$. Tumours from UK patients with squamous carcinoma who survived were significantly less likely to be E-cadherin absent than those from Chinese patients with squamous carcinomas who survived $(\mathbf{p}=\mathbf{0 . 0 0 7})$. Multivariate analysis $(n=37$ UK, paired data) showed that absence of E-cadherin in the primary tumour was a weak independent prognostic factor for non-survival $(30 \%$ significance level; $p=0.26$; odds ratio $=3.56$ ). In UK nodal metastases there was no correlation between E-cadherin expression and survival.
Conclusions-Squamous carcinomas from UK patients differed from both adenocarcinomas from UK patients and carcinomas from Chinese patients with respect to E-cadherin expression and prognostic significance. In tumours from UK patients, E-cadherin absence in the primary carcinoma (a weak independent prognostic factor) but not metastases correlated with non-survival.

(F Clin Pathol 1997;50:640-644)

Keywords: cadherins; China; oesophageal neoplasms

In some areas of China, the annual mortality rate from oesophageal cancer is 133 per 100000 people. ${ }^{1}$ Rates in the UK and many Western countries range from 2-15 per 100000 people. ${ }^{1}$ Survival of UK patients with squamous carcinoma (the almost exclusive histological type found in China) is better than that of those with adenocarcinoma. Survival is better for women but declines with age. ${ }^{2}$ Oesophageal cancer has a high metastatic potential and poor prognosis. The majority of patients has disseminated disease at presentation. $^{3}$

E-cadherin is a transmembrane, cell-cell, homophilic adhesion molecule present at zonulae adherentes of all epithelia and is essential for cell polarity and differentiation. In malignancy, E-cadherin may act as an antimetastasis molecule..$^{4-9}$ Downregulation of E-cadherin expression has been shown to be inversely correlated with differentiation and advanced stage in bladder, ${ }^{10}$ breast, ${ }^{11} 12$ cervical, ${ }^{13}{ }^{14}$ colorectal, ${ }^{15}$ endometrial, ${ }^{16}$ gastric, ${ }^{17}$ kidney, ${ }^{18}$ lung, ${ }^{19}$ pancreatic, ${ }^{20}$ and prostate ${ }^{21}$ cancers.

However, there have been few previous studies of E-cadherin in vivo in oesophageal cancer. Material from small numbers of tumours ${ }^{82-26}$ or cell lines ${ }^{27}$ only have been used. In addition, E-cadherin expression has been related to stage and grade of tumours only, rather than to survival. The present study investigated E-cadherin expression in an archival series of UK and Chinese patients with a five year postoperative survival, compared with that in 
Table 1 Patient details and histopathological staging and grading of resected tumours

\begin{tabular}{|c|c|c|c|c|c|c|c|c|c|c|c|c|}
\hline & $\begin{array}{l}\text { Mean age } \\
\text { (range) }\end{array}$ & $\begin{array}{l}\text { Sex } \\
(M / F)\end{array}$ & $T 1$ & $T 2$ & $T 3$ & $T 4$ & NO & $N 1$ & $N X$ & $G 1$ & $G 2$ & G3 \\
\hline \multicolumn{13}{|l|}{ Group 1} \\
\hline 17 UK adenocarcinoma survivors & $\begin{array}{l}66.8 \\
(46-78)\end{array}$ & $16 / 1$ & 6 & 1 & 10 & 0 & 12 & 5 & 0 & 0 & 10 & 7 \\
\hline $17 \mathrm{UK}$ adenocarcinoma non-survivors & $\begin{array}{l}60.8 \\
(45-75)\end{array}$ & $16 / 1$ & 0 & 1 & 15 & 1 & 1 & 14 & 2 & 0 & 2 & 15 \\
\hline \multicolumn{13}{|l|}{ Group 2} \\
\hline 23 UK squamous carcinoma survivors & $\begin{array}{l}56.9 \\
(31-76)\end{array}$ & $6 / 17$ & 3 & 2 & 18 & 0 & 14 & 9 & 0 & 0 & 13 & 10 \\
\hline $\begin{array}{l}23 \text { UK squamous carcinoma } \\
\text { non-survivors }\end{array}$ & $\begin{array}{l}63.8 \\
(55-72)\end{array}$ & $6 / 17$ & 1 & 0 & 21 & 1 & 10 & 12 & 1 & 0 & 7 & 16 \\
\hline \multicolumn{13}{|l|}{ Group 3} \\
\hline $\begin{array}{l}30 \text { Chinese squamous carcinoma } \\
\text { survivors }\end{array}$ & $\begin{array}{l}57.8 \\
(44-72)\end{array}$ & $18 / 12$ & 1 & 11 & 18 & 0 & 14 & 0 & 16 & 0 & 10 & 20 \\
\hline $\begin{array}{l}30 \text { Chinese squamous carcinoma } \\
\text { non-survivors }\end{array}$ & $\begin{array}{l}66.4 \\
(54-79)\end{array}$ & $18 / 12$ & 0 & 8 & 22 & 0 & 10 & 15 & 5 & 1 & 2 & 27 \\
\hline
\end{tabular}

matched patients who died of their tumour within five years.

\section{Methods}

Localised tumours were resected from $80 \mathrm{UK}$ (1977-89) and 60 Chinese (1979-88) patients. Cases were matched into two national groups where a five year survivor was paired with a non-survivor by matching tumour type (squamous carcinoma or adenocarcinoma), sex, and as closely as possible, age, and year of operation. Cause of death was confirmed as oesophageal cancer for the non-survivor group. None of the cases died within 30 days of surgery. Seventeen UK adenocarcinoma survivors, 23 UK squamous carcinoma survivors, and 30 Chinese squamous carcinoma survivors were paired with equal numbers of matched non-survivors, giving groups 1, 2, and 3, respectively. Patient details and staging of resected tumours are given in table 1.

Sections $(3 \mu \mathrm{m})$ were cut from archival wax blocks of primaries and nodal metastases (where available). E-cadherin was detected immunohistochemically, following microwave treatment $(0.01 \mathrm{M}$ citrate buffer, $\mathrm{pH} 6$, at $650 \mathrm{~W}$ for three five-minute cycles), using Vectastain Elite ABC and DAB Peroxidase Substrate Kits (Vector Laboratories, Peterborough, UK). The primary antibody was anti-Ecadherin (mouse monoclonal antihuman, Clone HECD-1; R \& D Systems, Abingdon, $\mathrm{UK}$ ), diluted $1 / 100$ in phosphate buffered saline (PBS), $\mathrm{pH} 7.4$; incubation was for 30 minutes at room temperature. Positive and negative controls were parallel sections of normal colon in PBS, with and without primary antibody, respectively.

Distribution of E-cadherin was classified as membranous, membranous and cytoplasmic or cytoplasmic. Staining was classified as positive if $>90 \%$ of cells were stained, heterogeneous if $10-90 \%$ were stained, and negative if $<10 \%$ were stained. Staining intensity was ignored. Staining was assessed independently by two observers with no knowledge of survival status. Consensus was achieved easily.

Histological type, staging and grade (G) were reported according to the tumour, node, metastasis ( $\mathrm{pTNM}$ ) classification of the UICC. ${ }^{28}$

\section{UNIVARIATE STATISTICAL ANALYSIS}

Analysis of matched primary tumours of survivors and non-survivors for E-cadherin in all three groups, and group 1 combined with group 2 used McNemar's test of symmetry. ${ }^{29}$ Comparison of groups 2 and 3 and investigation of associations between E-cadherin and staging variables in the UK and Chinese groups used $\chi^{2}$ tests, or Fisher's exact test ${ }^{29}$ where frequencies were small. Secondary nodal metastases were analysed using Fisher's exact test.

\section{MULTIVARIATE STATISTICAL ANALYSIS}

Multivariate statistical analysis was performed on group 1 plus group 2: 37 survivors/37 nonsurvivors with complete data. Logistic regression for 1-1 matched data ${ }^{30}$ analysed E-cadherin, T, N, and $\mathrm{G}$ by a stepwise selection of the significant explanatory variables for survival (SAS software, The SAS Institute, Cary, North Carolina, USA).

\section{Results}

Normal squamous oesophageal epithelium had a banded pattern of E-cadherin expression (fig 1). All layers, except negatively stained flattened squames, showed a membranous staining pattern, with some cytoplasmic staining in basal and parabasal layers. Normal glandular epithelium showed membranous staining with some cytoplasmic staining in the proliferative region. Dysplastic squamous (fig 2) and glandular epithelia showed weakened membranous staining and increased cytoplasmic expression, with negative basal layers in many cases. There was no difference in expression between UK and Chinese epithelia. When reporting tumours, E-cadherin in normal epithelia acted as an internal positive control.

Results of staining of primary tumours are shown in table 2 . Only one of 140 tumours had a positive, membranous staining pattern (from a Chinese survivor). Membranous staining was seen most often in the better differentiated areas, with cytoplasmic staining, or negative staining, in the less well differentiated areas. An area from an adenocarcinoma with a positive, membranous staining pattern is shown in fig 3 . Nodal metastases were assessed for E-cadherin (table 2) expression. Two of 54 metastases (from one UK adenocarcinoma survivor and 


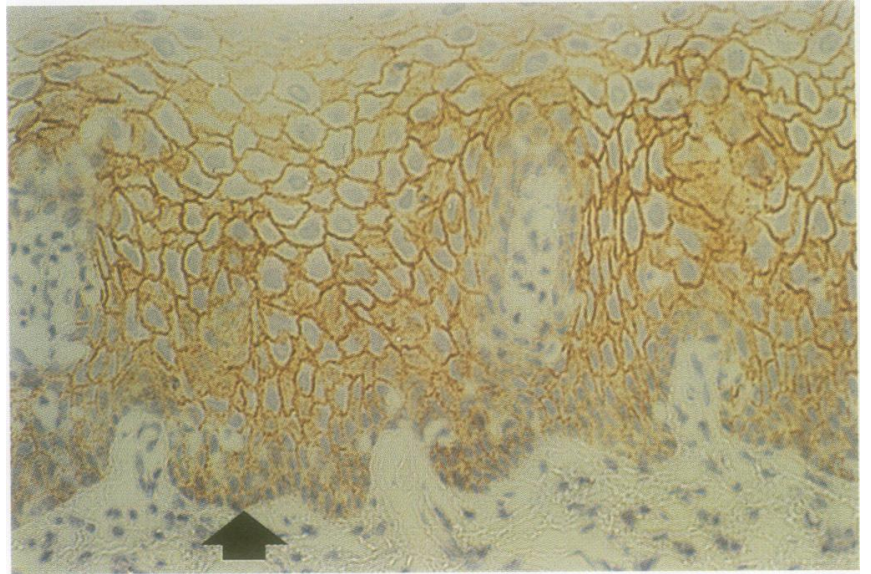

Figure 1 E-cadherin immunoreactivity of normal oesophageal squamous epithelium. Basal (arrowed) and parabasal cells show cytoplasmicl membranous staining. Prickle cell membranes are stained, with expression lessening as the cells approach the lumen.

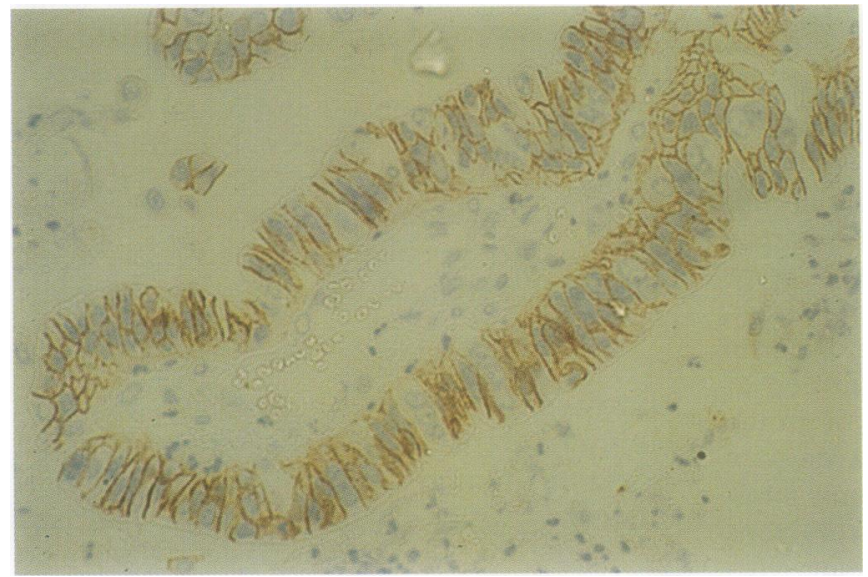

Figure 3 Membranous $E$-cadherin staining of a well differentiated area of an adenocarcinoma; $U K$ survivor.

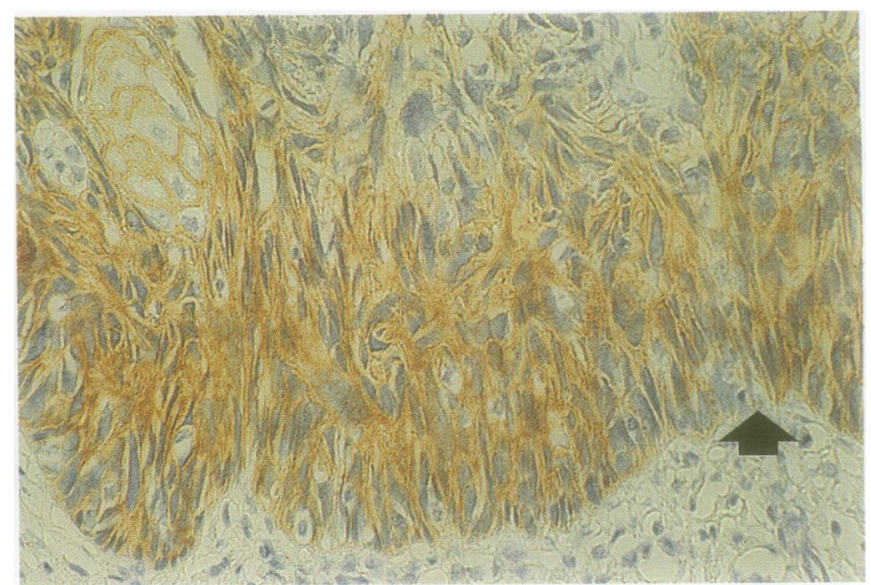

Figure 2 E-cadherin immunoreactivity of dysplastic oesophageal squamous epithelium is restricted to cytoplasmic staining (basal layer arrowed).

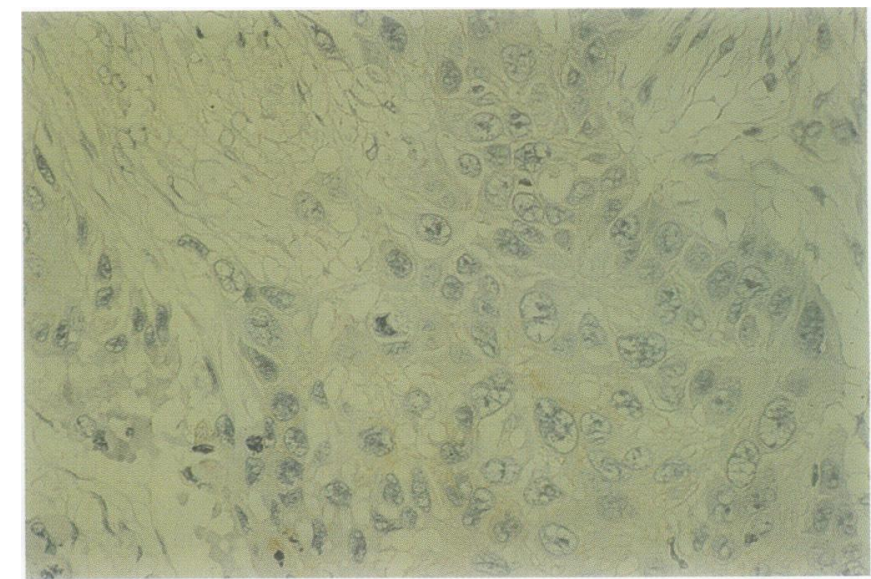

Figure 4 Primary squamous carcinoma negative for E-cadherin; UK non-survivor. one UK adenocarcinoma non-survivor) had positive, membranous staining. Numbers were small but by inspection any change in expression from that of the primary tumour to that of a derived secondary was variable. In UK survivors, 12 maintained the same expression, one had decreased expression, and one increased expression. In UK non-survivors, 19 maintained the same expression, two had decreased expression, and four had increased expression; in Chinese non-survivors, six maintained the same expression, four had decreased expression, and five had increased expression (data not shown). Comparison can be made between a squamous negatively expressing primary (fig

Table 2 Results of E-cadherin expression (present/absent) of primary tumours and nodal metastases

\begin{tabular}{|c|c|c|c|c|}
\hline & \multicolumn{2}{|l|}{ Tumours } & \multicolumn{2}{|c|}{ Nodal metastases } \\
\hline & Present & Absent & Present & Absent \\
\hline \multicolumn{5}{|l|}{ Group 1} \\
\hline 17 UK adenocarcinoma survivors & 16 & 1 & 3 & 2 \\
\hline 17 UK adenocarcinoma non-survivors & 16 & 1 & 13 & 1 \\
\hline \multicolumn{5}{|l|}{ Group 2} \\
\hline 23 UK squamous carcinoma survivors & 23 & 0 & 9 & 0 \\
\hline 23 UK squamous carcinoma non-survivors & 16 & 7 & 8 & 3 \\
\hline \multicolumn{5}{|l|}{ Group 3} \\
\hline 30 Chinese squamous carcinoma survivors & 22 & 8 & - & - \\
\hline $\begin{array}{l}30 \text { Chinese squamous carcinoma } \\
\text { non-survivors }\end{array}$ & 14 & 16 & 8 & 7 \\
\hline
\end{tabular}

*One of 12 nodal metastases not assessable for E-cadherin because of insufficient tissue.
4) and its derived positively expressing secondary (fig 5).

\section{UNIVARIATE STATISTICAL ANALYSIS}

Analysis showed a disparity in results from the primary tumours. In group 1 (UK adenocarcinoma) there was no correlation between the absence of E-cadherin and non-survival $(p=1.00)$. However, in group 2 (UK squamous carcinoma) tumours from nonsurvivors were significantly more likely not to express E-cadherin $(p=0.009)$. In group 3 (Chinese squamous carcinoma), there was no correlation between the absence of E-cadherin and non-survival $(p=0.06)$. Comparison of group 2 with group 3 showed that tumours from UK squamous survivors were significantly less likely to be E-cadherin absent than those from Chinese survivors $(p=0.007)$, and in the non-survivors, the tumours from Chinese patients had a greater proportion of E-cadherin absent $(p=0.10)$. When considering all UK cancers (group 1 plus group 2), absence of E-cadherin significantly correlated with non-survival $(p=0.02)$. When E-cadherin was absent, UK and Chinese cancers were significantly more likely to be G3 ( $p=0.08$ and $p=0.04$, respectively). In the UK nodal metastases there was no association between absence of E-cadherin and nonsurvival. 


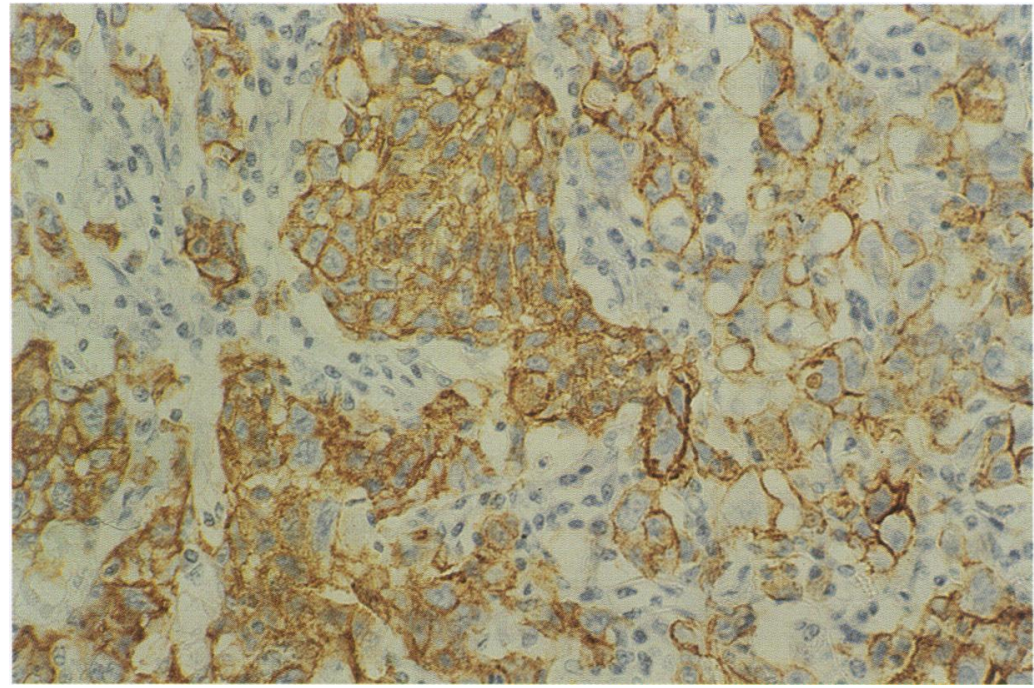

Figure 5 Nodal metastasis positive for $E$-cadherin, derived from the negative primary squamous carcinoma shown in fig 4.

\section{MULTIVARIATE STATISTICAL ANALYSIS}

This analysis used data from group 1 plus group 2 (37 UK matched pairs: three NX cases not analysed). Using a 5\% significance level, N1 rather than N0 ( $p=0.01$; odds ratio $=4.97)$ followed by $T 3 / 4$ rather than $T 1 / 2$ status $(p=0.03$; odds ratio $=11.64)$ were selected for non-survival.

It was not until a $30 \%$ significance level was chosen that E-cadherin was brought into the model as a third significant explanatory variable for non-survival. N1 rather than N0 $(\mathrm{p}=0.04$; odds ratio $=3.83$ ) was followed by $\mathrm{T} 3 / 4$ rather than $\mathrm{T} 1 / 2$ ( $\mathrm{p}=0.04$; odds ratio $=10.55)$, and then E-cadherin $(p=0.26$; odds ratio $=3.56$ ). A patient with an E-cadherin absent tumour was approximately four times more likely to be a non-survivor compared with a patient with an E-cadherin present tumour.

\section{Discussion}

Pairing survivors and non-survivors eliminated tumour type, age, and sex as prognostic factors. Presumably, survivors had less aggressive tumours, although four UK survivors did die of recurrent disease. Demographics and staging of the groups were well matched except for the disparity in tumour types. Adenocarcinomas are rare in China and were not represented. Squamous carcinomas found in China and the West may have different aetiologies, alcohol and tobacco being major determinants in the West but diet and other environmental factors being more important in China. However, once cancers have developed, there do not appear to be significant differences in the inherent behaviour of the tumours. ${ }^{1}$

In normal oesophageal epithelium, the least differentiated but more mobile cells have cytoplasmic staining for E-cadherin, with development of membranous staining as cells differentiate. Loss of expression by squames could permit desquamation. Dysplastic epithelia showed cytoplasmic staining, or a negative pattern, probably in association with poorer differentiation (also reported previously in dysplastic Barrett's oesophagus). ${ }^{85}{ }^{26}$ In oesopha- geal tumours, membranous staining was associated with better differentiation and cytoplasmic or negative staining with poorer differentiation. When present, a cytoplasmic or negative staining pattern is an early event in oesophageal dysplasia/tumorigenesis.

The disordered E-cadherin expression seen in the majority of oesophageal carcinomas could help to explain their high metastatic potential, as probably it reflects a reduction or loss of cell-cell adhesion (as has been demonstrated in squamous cell lines). ${ }^{27}$ Several authors ${ }^{9}$ have considered that cytoplasmic E-cadherin must be non-functional, cell adhesion and polarity requiring a membranous site. Jankowski et al suggest that there may be molecular alterations in E-cadherin in Barrett's oesophagus and adenocarcinoma. ${ }^{8}$

It is of particular interest that a significant relation between E-cadherin absence and nonsurvival exists for UK squamous carcinomas but not for UK adenocarcinomas or Chinese squamous carcinomas. Adenocarcinomas and squamous carcinomas are known to differ biologically (in terms of both the transversion/ transition spectrum of mutations ${ }^{31}$ and the immunohistochemical expression ${ }^{32}$ of the tumour suppressor gene, p53) and, therefore, they may have different aetiologies: the former are also more aggressive clinically. ${ }^{2}$ In survivors and non-survivors from the UK and China there were also discrepancies in E-cadherin status. Perhaps the squamous carcinomas found in China (high risk) and the West (low risk) are different disorders in terms of expression of E-cadherin and aetiology. Mutation of p53 is also known to be variable in squamous oesophageal tumours; in high risk areas of China, expression is $87.2 \%{ }^{33}$ in lower risk areas of China $64 \%,{ }^{33}$ in Germany $67.4 \%,{ }^{34}$ and in the UK $64 \% .^{32}$ Expression of p53 is a significant prognostic indicator in Chinese ${ }^{33}$ but not in European cancers. ${ }^{32}{ }^{34}$ E-cadherin loss and other accumulated genetic events may have different roles to play in the tumorigenesis and metastasis of these three distinct oesophageal malignancies.

E-cadherin expression in the primary, not the secondaries, is the factor relating to survival. Comparison of E-cadherin of an individual primary with its derived secondary gave variable results; the latter had predominantly the same expression but expression could either be reduced or increased. Previous reports have found E-cadherin expression in metastases equal to, or often greater than, the primary, ${ }^{25}$ abnormal or absent, ${ }^{8}$ or reduced in tumours with extensive nodal metastases. ${ }^{23} 24$ Differences in expression between primary and secondary tumours could reflect a primary heterogeneity, or instability of E-cadherin expression. Abnormality might allow detachment from the primary followed by reexpression and adhesion in the secondary site, perhaps under local influences. ${ }^{9}$

Multivariate analysis of UK primary tumours showed absence of E-cadherin was a weak prognostic indicator for non-survival, behind node positivity and increased depth of invasion but ahead of grade. E-cadherin is only 
one factor in cell-cell, cell-matrix adhesion. A single abnormality in a complex system might be unlikely to have an independent prognostic effect. Further studies of other factors, such as catenins ${ }^{9}{ }^{23}$ may lead to a better understanding of tumorigenesis and metastasis.

WJ, SJD and KJ are supported by the Oesophageal Cancer Fund, Birmingham UK. We are grateful to Dr Peijing Wu of the Department of Pathology, Shan Xian Central Hospital, Peoples's Republic of China, for providing a proportion of the archival pathological material and clinical data on Chinese cases. We thank Miss J Oates for invaluable technical assistance. R\&D Systems Europe Ltd provided a grant towards the cost of publication of colour figures.

1 Lewin K.J, Dawsey SM, Wang GQ. Squamous carcinoma of the esophagus in China and the West: are they different disorders? Dis Esophagus 1995;8:44-7.

2 Matthews HR, Waterhouse JAH, Powell J, McConkey CC, Robertson JE, eds. Overall survival. In: Clinical cancer Robertson JE, eds. Overall survival. In: Clinical cancer
monographs. Vol 1. Cancer of the oesophagus. London: Macmonographs. Vol 1. Cancer
millan, 1987:62, 68, 78 .

3 Kelsen DP. More or less surgery for esophageal cancer: non-operative therapy of esophageal cancer. Dis Esophagu 1994;7:162-8.

4 Takeichi $M$. The cadherins: cell-cell adhesion molecules controlling animal morphogenesis. Development 1988;102 639-55.

5 Pignatelli M. E-cadherin: a biological marker of tumour differentiation [editorial]. F Pathol 1993;171:81-2.

6 Behrens J. Cadherins as determinants of tissue morphology and suppressors of invasion. Acta Anat 1994;149:165-9.

7 Jiang WG, Puntis MCA, Hallett MB. Molecular and cellular basis of cancer invasion and metastasis: implications for lar basis of cancer invasion and metasta
treatment. Br $\mathcal{F}$ Surg 1994;81:1576-90.

treatment. Br 7 Surg 1994;81:1576-90.
8 Jankowski JA, Newham PM, Kandemir O, Hirano S Takeichi M, Pignatelli $M$. Differential expression of E-cadherin in normal, metaplastic and dysplastic oesophageal mucosa: a putative biomarker. Int $\mathcal{F}$ Oncol 1994;4:4418.

9 Shiozaki $\mathrm{H}$, Oka $\mathrm{H}$, Inoue $M$, Tamura $S$, Monden $M$ E-cadherin mediated adhesion system in cancer cells. Cancer 1996;77:1605-13.

10 Bringuier PP, Umbas R, Schaafsma HE, Karthaus HFM Debruyne FMJ, Schalken JA. Decreased E-cadherin immunoreactivity correlates with poor survival in patients immunoreactivity correlates with poor survival in p

11 Gamallo C, Palacios J, Suarez A, Pizarro A, Navarro P, Quintanilla $\mathrm{M}$, et al. Correlation of E-cadherin expression with differentiation grade and histological type in breast carcinoma. Am F Pathol 1993;142:987-93.

12 Rasbridge SA, Gillett CE, Sampson SA, Walsh FS, Millis RR. Epithelial (E-) and placental (P-) cadherin cell adhesion molecule expression in breast carcinoma. 7 Pathol 1993;169:245-250.

13 Schipper JH, Frixen UH, Behrens J, Unger A, Jahnke K, Birchmeir W. E-cadherin expression in squamous cell carcinomas of head and neck: inverse correlation with tumor dedifferentiation and lymph node metastasis. Cancer Res 1991;51:6328-37.

14 Vessey CJ, Wilding J, Folarin N, Hirano S, Takeichi M, Soutter $\mathrm{P}$, et al. Altered expression and function of E-cadherin in cervical intraepithelial neoplasia and invasive squamous cell carcinoma. F Pathol 1995;176:151-9.

15 Nigam AK, Savage FJ, Boulos PB, Stamp GWH, Liu D, Pignatelli $M$. Loss of cell-cell and cell-matrix adhesion molecules in colorectal cancer. Br $\mathcal{F}$ Cancer 1993;68:50714.

16 Sakuragi N, Nishiya M, Ikeda K, Ohkouch T, Furth EE, Hareyama $\mathrm{H}$, et al. Decreased E-cadherin expression in endometrial carcinoma is associated with tumor dedifferentiation and deep myometrial invasion. Gynecologic Oncol 1994;53:183-189.

17 Mayer B, Johnson JP, Leitl F, Jauch KW, Heiss MM, Schildberg FW, et al. E-cadherin expression in primary and metastatic gastric cancer: down-regulation correlates with celluar dedifferentiation and glandular disintegration. Cancer Res 1993;53:1690-5.

18 Katagiri A, Watanabe R, Tomita Y. E-cadherin expression in renal cell cancer and its significance in metastasis and survival. Br f Cancer 1995;71:376-9.

19 Böhm M, Totzeck B, Birchmeir W, Wieland I. Differences of E-cadherin expression levels and patterns in primary and metastatic human lung cancer. Clin Exp Metastasis 1994;12:55-62.

20 Pignatelli M, Ansari TW, Gunter P, Liu D, Hirano S, Takeichi $M$, et al. Loss of membranous E-cadherin expression in pancreatic cancer: correlation with lymph node metastasis, high grade, and advanced stage. 7 Pathol 1994; metastasis,

21 Otto T, Rembrink K, Goepel M, Meyer-Schwickerath M, Rübben H. E-cadherin: a marker for differentiation and invasiveness in prostatic carcinoma. Urol Res 1993;21:35962 .

22 Shiozaki $\mathrm{H}$, Tahara $\mathrm{H}$, Oka $\mathrm{H}$, Miyata $\mathrm{M}$, Kobayashi $\mathrm{K}$, Tamura S, et al. Expression of immunoreactive E-cadherin adhesion molecules in human cancers. Am $\mathcal{f}$ Pathol 1991;139:17-23.

23 Kadowaki $T$, Shiozaki $H$, Inoue $M$, Tamura S, Oka H, Doki $\mathrm{Y}$, et al. E-cadherin and $\alpha$-catenin expression in human esophageal cancer. Cancer Res 1994;54:291-6.

24 Miyata $M$, Shiozaki $H$, Iihara $K$, Shimaya K, Oka $H$, Kadowaki $\mathrm{T}$, et al. Relationship between E-cadherin expression and lymph node metastasis in human esophageal cancer. Int f Oncol 1994;4:61-5.

25 Bongiorno PF, Al-Kasspooles M, Lee SW, Rachwal WJ, Moore JH, Whyte RI, et al. E-cadherin expression in primary and metastatic thoracic neoplasms and in Barrett's oesophagus. Br F Cancer 1995;71:166-72.

26 Swami S, Kumble S, Triadafilopoulos G. E-cadherin expression in gastroesophageal reflux disease, Barrett's esophagus, and esophageal adenocarcinoma: an immunohistochemical and immunoblot study. Am $\mathcal{f}$ Gastroenterol 1995;90:1808-13.

27 Doki Y, Shiozaki H, Tahara H, Inoue M, Oka H, Iihara K, et al. Correlation between E-cadherin expression and invasiveness in vitro in a human esophageal cancer cell line. Cancer Res 1993;53:3421-6.

28 Hermanek P, Sobin LH, eds. UICC International Union against Cancer. TNM classification of malignant tumours. 4th edn. 2nd rev. Berlin: Springer Verlag, 1992:42-4

29 Everitt BS. The analysis of contingency tables. London: Chapman and Hall, 1977.

30 Hosmer DW, Lemeshow S. Applied logistic regression. New York: John Wiley and Sons, 1989.

31 Gleeson CM, Sloan JM, McGuigan JA, Ritchie AJ, Russell SEH. Base transitions at $\mathrm{CpG}$ dinucleotides in the $\mathrm{p} 53$ gene are common in esophageal adenocarcinoma. Cancer gene are common

32 Vijeyasingam R, Darnton SJ, Jenner $\mathrm{K}$, Allen CA, Billingham C, Matthews HR. Expression of p 53 protein in oesophageal carcinoma: clinicopathological correlation and prognostic significance. Br f Surg 1994;81:1623-6.

33 Wang D-Y, Xiang Y-Y, Tanaka M, Li X-R, Li J-R, Shen Q, et al. High prevalence of $\mathrm{p} 53$ protein overexpression in patients with esophageal cancer in Linxian, China and its relationship to progression and prognosis. Cancer 1994;74: 3089-96.

34 Sarbia M, Porschen R, Borchard F, Horstmann O, Willers $\mathrm{R}$, Gabbert HE. p 53 protein expression and prognosis in squamous cell carcinoma of the esophagus. Cancer $1994 ; 74: 2218-23$ 